\title{
WHEN POLARIZATIONS GENERATE
}

\author{
GERALD W. SCHWARZ
}

\begin{abstract}
Let $G$ be a reductive complex algebraic group and $V$ a finitedimensional $G$-module. From elements of the invariant algebra $\mathbb{C}[V]^{G}$ we obtain by polarization elements of $\mathbb{C}[k V]^{G}$, where $k \geq 1$ and $k V$ denotes the direct sum of $k$ copies of $V$. For $G$ simple our main result is the classification of the $G$-modules $V$ and integers $k \geq 2$ such that polarizations generate $\mathbb{C}[k V]^{G}$.
\end{abstract}

\section{INTRODUCTION}

Our base field is $\mathbb{C}$, the field of complex numbers. Throughout this paper, $G$ will denote a reductive algebraic group. All our $G$-modules are assumed to be finitedimensional and rational. Let $V$ be a $G$-module and let $f \in \mathbb{C}[V]^{G}$ be homogeneous of degree $d$. For $v_{1}, v_{2}, \ldots, v_{k} \in V$, consider the function $f\left(\sum_{i} s_{i} v_{i}\right)$ where the $s_{i}$ are indeterminates. Then

$$
f\left(\sum_{i} s_{i} v_{i}\right)=\bigoplus_{\alpha \in\left(\mathbb{Z}^{+}\right)^{k},|\alpha|=d} s^{\alpha} f_{\alpha}\left(v_{1}, \ldots, v_{k}\right)
$$

where the $f_{\alpha} \in \mathbb{C}[k V]^{G}$ are multihomogeneous of the indicated degrees $\alpha$. Here for $\alpha=\left(a_{1}, \ldots, a_{k}\right) \in\left(\mathbb{Z}^{+}\right)^{k}$ we have $s^{\alpha}=s_{1}^{a_{1}} \cdots s_{k}^{a_{k}}$ and $|\alpha|=a_{1}+\cdots+a_{k}$. We call the $f_{\alpha}$ the polarizations of $f$. Let $\operatorname{pol}_{k}(V)^{G}$ denote the subalgebra of $\mathbb{C}[k V]^{G}$ generated by polarizations. We say that $V$ has the $k$-polarization property if $\operatorname{pol}_{k}(V)^{G}=\mathbb{C}[k V]^{G}$.

For $G=\mathrm{O}_{n}$ and $V$ the standard action on $\mathbb{C}^{n}$ one has the $k$-polarization property for all $k$. We have the same result when $G=S_{n}$ and $V$ is the standard action on $\mathbb{C}^{n}$ by WeYL Weyl46 and also for the standard actions of the Weyl groups of type $B$ and $C$ by Hunziker Hun97. From Wallach Wal93 we learn that the 2-polarization property is false for the Weyl group of type $D_{4}$.

One can also ask if $\mathbb{C}[k V]^{G}$ is finite over $\operatorname{pol}_{k}[V]^{G}$. In case $V$ is a module for $\mathrm{SL}_{2}$ which does not contain a copy of the irreducible two-dimensional module, then $\mathbb{C}[k V]^{G}$ is finite over $\operatorname{pol}_{k}[V]^{G}$ for all $k$. See Kraft-Wallach KrWa04 ( $V$ irreducible) and Losik-Michor-Popov [LMP06 for the general case. The same references prove the $k$-polarization property for all $k$ when $G$ is a torus. However, for the adjoint representation of a simple group of rank at least 2, finiteness fails for $k \geq 2$ [LMP06. See Draisma-Kemper-Wehlau DKW06 for questions about polarization and separation of orbits.

Example 1.1. Let $V:=\mathbb{C}^{2}$ be the two-dimensional irreducible $G:=\mathrm{SL}_{2}$-module. Then $\mathbb{C}[V \oplus V]^{G}$ is generated by the determinant function. The polarizations of this generator give rise to three of the six determinant generators of $\mathbb{C}[4 V]^{G}$. Hence

Date: September 2006.

2000 Mathematics Subject Classification. 14L30, 20C15, 20 G20.

Partially supported by NSA Grant H98230-06-1-0023. 
$V \oplus V$ does not have the 2-polarization property. Of course, neither does $V$, since $\mathbb{C}[V]^{G}=\mathbb{C}$ while $\mathbb{C}[V \oplus V]^{G} \neq \mathbb{C}$.

Our main aim is to classify the $G$-modules which have the $k$-polarization property, $k \geq 2$, when $G$ is a simple linear algebraic group. Along the way we establish general criteria for a representation to have the $k$-polarization property. Note the trivial fact that the $(k+1)$-polarization property fails if the $k$-polarization property fails. The crucial case to consider is usually that of $k=2$.

We thank V. Popov for useful comments.

\section{SLICES}

We establish some tools for obtaining our classification. Let $V$ be a $G$-module and let $f \in \mathbb{C}[V]^{G}$ be homogeneous of degree $d$. If $k=2$ or 3 , then we will denote the polarizations of $f$ as $\left\{f_{i, j}\right\}_{i+j=d}$ and $\left\{f_{i, j, k}\right\}_{i+j+k=d}$, respectively. We leave the proof of the following to the reader.

Lemma 2.1. Let $V$ be a $G$-module with the $k$-polarization property. Then any $G$-submodule of $V$ has the k-polarization property.

Let $V$ be a $G$-module. Then $\mathbb{C}[V]^{G}$ is finitely generated, and we denote by $\pi: V \rightarrow V / / G$ the morphism of affine varieties dual to the inclusion $\mathbb{C}[V]^{G} \subset \mathbb{C}[V]$.

Let $V$ be a $G$-module and $v \in V$ such that the orbit $G \cdot v$ is closed. Then the isotropy group $G_{v}$ is reductive and there is a splitting $V=S \oplus T_{v}(G \cdot v)$ of $G_{v}$-modules. The representation $G_{v} \rightarrow \mathrm{GL}(S)$ is called the slice representation at $v$. We can arrange that $v \in S$. We have a canonical map $\varphi: G *^{G_{v}} S \rightarrow G \cdot S$ which is equivariant. Here $G *^{G_{v}} S$ is the quotient of $G \times S$ by the $G_{v}$-action sending $(g, s) \mapsto\left(g h^{-1}, h \cdot s\right)$ for $h \in G_{v}, g \in G$ and $s \in S$. The $G_{v}$-orbit of $(g, s)$ is denoted $[g, s]$. Then $\varphi: G *^{G_{v}} S \rightarrow V$ sends $[g, s]$ to $g(v+s)$. Replacing $S$ by an appropriate $G_{v}$-stable neighborhood of $0 \in S$ one has Luna's slice theorem Lu73. But here we only need one consequence of this theorem. Namely, that the induced mapping $\varphi / / G:\left(G *^{G_{v}} S\right) / / G \simeq S / / G_{v} \rightarrow V / / G$ induces an isomorphism of the Zariski cotangent spaces at the points 0 and $G \cdot v$ in the quotients.

Lemma 2.2. Let $v, G_{v}$, etc. be as above. Suppose that $V$ has the $k$-polarization property. Then so does the $G_{v}$-module $S$.

Proof. We treat the case $k=2$ and leave the general case to the reader. A $G_{v}$-stable complement to $T_{(v, 0)}(G \cdot(v, 0))$ in $V \oplus V$ is $S \oplus V$. Let $I:=\left\{f \in \mathbb{C}[V \oplus V]^{G}: f(v, 0)=\right.$ $0\}$, let $J:=\left\{f \in \mathbb{C}[S \oplus V]^{G_{v}}: f(0,0)=0\right\}$ and let $K:=\left\{f \in \mathbb{C}[S \oplus S]^{G_{v}}: f(0,0)=\right.$ $0\}$. Let $\psi: G *^{G_{v}}(S \oplus V) \rightarrow(V \oplus V)$ be the canonical map. Then, as indicated above, Luna's slice theorem implies that $\psi^{*}$ induces an isomorphism of $I / I^{2}$ with $J / J^{2}$, and clearly $J / J^{2} \rightarrow K / K^{2}$ is surjective. Thus $I \rightarrow K / K^{2}$ is surjective.

Let $f \in \mathbb{C}[V]^{G}$ be homogeneous of degree $d>0$. Then $f-f(v) \in I \cap \mathbb{C}[V]^{G}$ and its image in $K \cap \mathbb{C}[S]^{G_{v}}$ is the mapping sending $s \in S$ to $f(v+s)-f(v)$. We have $f(v+s)-f(v)=\sum_{i+j=d, j>0} f_{i, j}(v, s)$. Thus each homogeneous component $f_{i, j}(v, s), j>0$, lies in $K \cap \mathbb{C}[S]^{G_{v}}$. If we polarize in the second argument we obtain the collection of functions $\left\{f_{i, j, k}\left(v, s, s^{\prime}\right): j+k>0\right\} \subset K$ where $s, s^{\prime} \in S$. On the other hand, if we take the polarizations $f_{d-k, k}$ of $f$ where $k>0$, then they are in $I$ and their images in $K$ are sums of the elements of $\left\{f_{i, j, k}\left(v, s, s^{\prime}\right): k>0\right\}$. Thus the images of the $f_{i, j}-\delta_{0 j} f(v)$ in $K$ are polarizations of elements of $K \cap \mathbb{C}[S]^{G_{v}}$. Since $V$ has the 2-polarization property, the $f_{i, j}-\delta_{0 j} f(v)$ generate $I$ (as one varies 
$f$ ), hence $K / K^{2}$ is generated by the polarizations of elements of $K \cap \mathbb{C}[S]^{G_{v}}$. But functions in $K$ which span $K / K^{2}$ generate $\mathbb{C}[S \oplus S]^{G_{v}}$, so $S$ has the 2-polarization property.

Remarks 2.3. (1) The $G_{v}$-fixed part of $S$ plays no role. If $S^{\prime}$ is the sum of the nontrivial isotypic components of $S$, then the interesting fact is that $\mathbb{C}\left[S^{\prime} \oplus S^{\prime}\right]^{G_{v}}$ is generated by polarizations.

(2) Suppose that $\operatorname{dim} \mathbb{C}[V]^{G}=1$ and the representation is stable (i.e., there is a non-empty open set of closed orbits). Then for any closed non-zero orbit $G \cdot v$, the slice representation is trivial, so that the Lemma is of no help.

\section{REPRESENTATIONS WITHOUT THE POLARIZATION PROPERTY}

One can say that "most" representations do not have the 2-polarization property. This is born out by the following sequence of lemmas.

Lemma 3.1. Suppose that $V=V_{1} \oplus V_{2}$ where the $V_{i}$ are $G^{0}$-stable and the elements of $G$ preserve the $V_{i}$ or interchange them. For example, the $V_{i}$ could be the isotypic components corresponding to nontrivial irreducible $G^{0}$-modules. Suppose further that $\mathbb{C}\left[V_{1} \oplus V_{2}\right]^{G}$ has a minimal bihomogeneous generator $f$ of degree $(a, b)$ where $a b \geq 2$. Then $V$ does not have the 2-polarization property.

Proof. Let $v_{i}, v_{i}^{\prime}$ denote elements in $V_{i}, i=1,2$. Set $d=a+b \geq 3$. Consider the polarization $f_{d-2,2}$ of $f$. We can write $f_{d-2,2}\left(v_{1}, v_{2}, v_{1}^{\prime}, v_{2}^{\prime}\right)$ as a sum of terms $f^{2,0}+f^{1,1}+f^{0,2}$ where $f^{i, j}\left(v_{1}, v_{2}, v_{1}^{\prime}, v_{2}^{\prime}\right)$ has homogeneity $(i, j)$ in $v_{1}^{\prime}$ and $v_{2}^{\prime}$. The $f^{i, j}$ are $G^{\prime}$-invariant, where $G^{\prime}$ is the subgroup of $G$ preserving the $V_{i}$. Clearly, up to a scalar, $G$ leaves $f^{2,0}+f^{0,2}$ and $f^{1,1}$ invariant. Hence the functions are $G$-invariant (since their sum is $G$-invariant) and nonzero (since $d \geq 3$ ). Let $I$ denote the ideal of elements of $\mathbb{C}[2 V]^{G}$ vanishing at 0 . Suppose that $\alpha f^{1,1}+\beta\left(f^{2,0}+f^{0,2}\right) \in I^{2}$ for some $\alpha$ and $\beta$. We may assume that $f^{2,0} \neq 0$, i.e., that $a \geq 2$. Then evaluating at points $\left(v_{1}, v_{2}, v_{1}, 0\right)$ we see that $\beta f \in I^{2}$, hence $\beta=0$. Now one evaluates at points $\left(v_{1}, v_{2}, v_{1}, v_{2}\right)$ to see that $\alpha=0$. We have shown that $f^{1,1}$ and $f^{2,0}+f^{0,2}$ are linearly independent modulo $I^{2}$.

If $f^{1,1}$ is in the subalgebra generated by polarizations of elements of $\mathbb{C}[V]^{G}$, then $f^{1,1}$ is a sum of terms $q r_{\bullet-2,2}$ and $s t_{\bullet-1,1} u_{\bullet-1,1}$ for appropriate homogeneous $q, \ldots, u \in \mathbb{C}[V]^{G}$. Since $f^{1,1}$ is a minimal generator, our sum has to contain terms of the form $r_{d-2,2}$. Thus we may assume that $f^{1,1} \in r_{d-2,2}+I^{2}$ for some $r$. Restituting we see that $(a b) f \in\left(\begin{array}{l}d \\ 2\end{array}\right) r+I^{2} \cap \mathbb{C}[V]^{G}$. Hence we have that $r+I^{2}=c f+I^{2}$ for some $c \neq 0$. It follows that $f^{1,1} \in c\left(f^{1,1}+f^{2,0}+f^{0,2}\right)+I^{2}$ which implies that $f^{1,1}$ and $f^{2,0}+f^{0,2}$ are linearly dependent modulo $I^{2}$. This is a contradiction, hence $V$ does not have the 2-polarization property.

Lemma 3.2. Suppose that $G$ acts on $G^{0}$ by inner automorphisms and that $V$ is a $G$-module which contains an irreducible symplectic $G^{0}$-submodule $U$. Further suppose that $\mathbb{C}[U]^{G^{0}}$ has generators of even degree. Then $V$ does not have the 2-polarization property.

Proof. We may suppose that, as $G^{0}$-module, $V$ is the isotypic component of type $U$. A central torus of $G^{0}$ must act trivially on $U$, so we can reduce to the case that $G^{0}$ is semisimple. Set $H:=Z_{G}\left(G^{0}\right)$. Then $H$ is finite and $G=H G^{0}$ where $H \cap G^{0}=Z\left(G^{0}\right)$. Now $W:=\operatorname{Hom}(U, V)^{G^{0}}$ is an $H$-module (via the action of $H$ on 
$V$ ) and we have a canonical $G$-equivariant isomorphism of $V$ with $W \otimes U$ where the latter is naturally an $\left(H \times G^{0}\right) / Z\left(G^{0}\right) \simeq G$-module. Let $k$ be minimal such that $\mathrm{S}^{2 k}\left(W^{*}\right)^{H} \neq 0$. We have a copy of $\mathrm{S}^{2}\left(W^{*}\right) \subset \mathrm{S}^{2}\left(W^{*}\right) \otimes \wedge^{2}\left(U^{*}\right)^{G^{0}} \subset\left(W^{*} \otimes U^{*} \otimes\right.$ $\left.W^{*} \otimes U^{*}\right)^{G^{0}} \subset \mathrm{S}^{2}\left(2 V^{*}\right)^{G^{0}}$. Then there is a nonzero $f$ in the copy of $S^{2 k}\left(W^{*}\right)^{H}$ in $S^{k}\left(S^{2}\left(W^{*}\right)\right)$. Suppose that $f$ is a polynomial in polarizations of generators of $\mathbb{C}[V]^{G}$. We may assume that each generator lies in $\mathrm{S}^{\lambda}\left(W^{*}\right)^{H} \otimes \mathrm{S}^{\lambda}\left(U^{*}\right)^{G^{0}}$ for some partition $\lambda$ (so $\mathrm{S}^{\lambda}$ denotes the corresponding Schur component). Then $f$ must be a polynomial in polarizations of elements of the $\mathrm{S}^{\lambda}\left(W^{*}\right)^{H} \otimes \mathrm{S}^{\lambda}\left(U^{*}\right)^{G^{0}}$ where $\mathrm{S}^{\lambda}$ is a symmetric power. But then by minimality of $k$ and the assumption that generators of $\mathbb{C}[U]^{G^{0}}$ have even degree, we must have that $f$ itself is a polarization. But, by construction, $f$ restitutes to 0 , i.e., $f(v, v)=0, v \in V$, hence $f$ is not a polarization.

Let $R_{j}$ denote the irreducible $\mathrm{SL}_{2}$-module of dimension $j+1, j \in \mathbb{N}$.

Corollary 3.3. Suppose that $G^{0}=\mathrm{SL}_{2}$ and that $V$ is a $G$-module which contains a $G^{0}$-submodule $R_{j}$ where $j$ is odd. Then $V$ does not have the 2-polarization property.

Proof. For $j$ odd, $R_{j}$ is a symplectic representation of $\mathrm{SL}_{2}$. Moreover, $\pm I \in \mathrm{SL}_{2}$ act as \pm 1 on $R_{j}$, so that all elements of $\mathbb{C}\left[R_{j}\right]^{\mathrm{SL}_{2}}$ have even degree. Finally, all automorphisms of $\mathrm{SL}_{2}$ are inner. Thus we can apply Lemma 3.2

Now assume that $G^{0}=\mathbb{C}^{*}$. Let $\nu_{j}$ denote the irreducible $\mathbb{C}^{*}$-module with weight $j$. We denote by $m \nu_{j}$ the direct sum of $m$ copies of $\nu_{j}$. Assume that $V$ is a $G$ module such that the multiplicity of each $\nu_{j}$ is the same as that of $\nu_{-j}$ for all $j$. We say that $V$ is balanced and we let $q(V)$ denote half the number of nonzero weight spaces, counting multiplicity.

Proposition 3.4. Suppose that $G^{0}=\mathbb{C}^{*}$. Let $V$ be a balanced $G$-module with $q(V) \geq 2$. Then $V$ does not have the 2-polarization property.

Proof. First assume that there is a nonzero weight $j$ of multiplicity $m \geq 2$. Then the $\mathbb{C}^{*}$-submodule $m\left(\nu_{j} \oplus \nu_{-j}\right)$ is $G$-invariant, so that we may assume that it is all of $V$. We may then also assume that $j=1$. Set $V_{1}:=m \nu_{1}$ and $V_{2}:=m \nu_{-1}$. If $f$ is a minimal homogeneous generator of degree at least 3 , then we are done by Lemma 3.1 Thus we may suppose that all the minimal homogeneous generators of $\mathbb{C}\left[V_{1} \oplus V_{2}\right]^{G}$ have degree 2. Let $G^{\prime}:=Z_{G}\left(G^{0}\right)$. Then $V_{1}$ and $V_{2}$ are $G^{\prime}$-modules. Write $V_{1}=\oplus W_{i}$ where the $W_{i}$ are irreducible $G^{\prime}$-submodules, and similarly write $V_{2}=\oplus U_{j}$. Then the quadratic $G^{\prime}$-invariants correspond to pairs $W_{i}$ and $U_{j}$ such that $U_{j} \simeq W_{i}^{*}$. But $\mathbb{C}[V]^{G^{0}}$ has to be finite over $\mathbb{C}[V]^{G^{\prime}}$ and this forces that $V_{1} \simeq m W$ and $V_{2} \simeq m W^{*}$ for some irreducible one-dimensional representation $W$ of $G^{\prime}$. It follows that the image of $G^{\prime}$ in $\operatorname{GL}(V)$ is that of $G^{0}=\mathbb{C}^{*}$, so we may assume that $G^{\prime}=G^{0}$. If $G=G^{0}$, then one can easily see that there are more quadratic generators in $\mathbb{C}[2 V]^{G}$ than those coming from polarizations. If $G \neq G^{0}$, then $G$ is generated by $G^{0}$ and an element $\alpha$ such that $\alpha t \alpha^{-1}=t^{-1}$ for $t \in G^{0}$ and $\alpha^{2} \in G^{0}$. Now $\alpha^{2}$ is fixed under conjugation by $\alpha$ so that $\alpha^{2}= \pm 1$. For an appropriate basis $v_{1}, \ldots v_{m}$ of $V_{1}$ and $w_{1}, \ldots, w_{m}$ of $V_{2}$ we have that $\alpha\left(v_{i}\right)=w_{i}$ and $\alpha\left(w_{i}\right)= \pm v_{i}, i=1, \ldots, m$. If $\alpha^{2}=-1$, then one can see that quadratic invariants do not generate $\mathbb{C}[V]^{G}$. If $\alpha^{2}=1$, then we just have $m$-copies of the standard representation of $\mathrm{O}_{2}$. Since $m \geq 2$, one easily sees that there are more generators of $\mathbb{C}[V \oplus V]^{G}$ than polarizations. 
Now suppose that $V$ contains two different pairs of weights. Then we can assume that $V=m_{1}\left(\nu_{p} \oplus \nu_{-p}\right) \oplus m_{2}\left(\nu_{q} \oplus \nu_{-q}\right)$ as $\mathbb{C}^{*}$-module where $p$ and $q$ are relatively prime and $m_{1}, m_{2} \geq 1$. There is then clearly a bihomogeneous minimal $G$-invariant of degree $(a, b)$ where $a b \geq 2$, so that we can again apply Lemma 3.1

If $V$ is a $G$-module where $G^{0}$ is simple of rank 1 then we define $q(V)$ as before, relative to the action of a maximal torus.

Corollary 3.5. Suppose that $G^{0}$ is simple of rank 1 and that $V$ is a $G$-module with $q(V) \geq 3$. Then $V$ does not have the 2 -polarization property.

Proof. By Corollary 3.3 we may assume that, as $G^{0}$-module, $V$ is the direct sum of copies of $R_{j}, j$ even. Let $v \in V$ be a nonzero zero weight vector. Then the $G$-orbit though $w$ is closed with isotropy group a finite extension of $\mathbb{C}^{*}$ and slice representation $V^{\prime}$ where $q\left(V^{\prime}\right) \geq 2$. By Proposition 3.4. $\left(V^{\prime}, G_{v}\right)$ does not have the 2-polarization property, hence neither does $V$.

\section{The Main Theorem}

Recall that a $G$-module is called coregular if $\mathbb{C}[V]^{G}$ is a regular $\mathbb{C}$-algebra.

Proposition 4.1. Let $V$ be an irreducible representation of the simple algebraic group $G$. If $V$ is not coregular, then $V$ does not have the 2-polarization property.

Proof. The representations $R_{j}$ of $\mathrm{SL}_{2}$ which are not coregular have $q\left(R_{j}\right) \geq 3$, hence they do not have the 2-polarization property by Corollary 3.5 By $S$ ch78, Remark 5.2] we know that if $V$ is not coregular and the rank of $G$ is at least 2, then one of the following occurs

(1) There is a closed orbit $G \cdot v$ such that $G_{v}$ has rank 1 . Let $G_{v} \rightarrow \operatorname{GL}(S)$ be the slice representation. Then $S$ is balanced. If $G_{v}^{0}$ is simple, then $q(S) \geq 4$ and if $G_{v}^{0} \simeq \mathbb{C}^{*}$, then $q(S) \geq 2$.

(2) $V=\mathrm{S}^{3}\left(\mathbb{C}^{4}\right)$ and $G=\mathrm{SL}_{4}$.

By Proposition 3.4 and Corollary 3.5] any representation in (1) above does not have the 2-polarization property. Thus the only remaining case is (2). Here the laziest thing to do is to use the program LiE vL94 to compute some low degree invariants of one or two copies of $V$. The first generator of $\mathrm{S}^{*}\left(V^{*}\right)^{G}$ occurs in $\mathrm{S}^{8}\left(V^{*}\right)$ and the dimension of the fixed space is 1 . In $S^{2}\left(V^{*}\right) \otimes S^{6}\left(V^{*}\right)$ there is a two-dimensional space of invariants, so that $V$ does not have the 2-polarization property.

We would not have had to use LiE if the following could be established.

Conjecture 4.2. Let $H \subset \mathrm{GL}(V)$ where $H$ is finite and not generated by reflections. Then $V$ does not have the 2-polarization property.

In the following we use the notation of [Sch78] for the simple groups and their representations. We list such representations as pairs $(V, G)$.

Theorem 4.3. Let $V$ be an irreducible nontrivial representation of the simple algebraic group $G$. If $V$ has the 2-polarization property, then, up to (possibly outer) isomorphism, the pair $(V, G)$ is on the following list.

(1) $\left(\varphi_{1}, A_{n}\right), n \geq 2$.

(2) $\left(\varphi_{1}^{2}, A_{n}\right), n \geq 1$.

(3) $\left(\varphi_{2}, A_{n}\right), n \geq 4$. 
(4) $\left(\varphi_{1}, B_{n}\right), n \geq 2$ and $\left(\varphi_{1}, D_{n}\right), n \geq 3$.

(5) $\left(\varphi_{1}, G_{2}\right)$.

(6) $\left(\varphi_{3}, B_{3}\right)$.

(7) $\left(\varphi_{1}, E_{6}\right)$.

Proof. One can verify from Sch78 that all the listed representations have the 2polarization property. We must rule out all other cases. The list of coregular representations is due to KAC-POPOV-VInBERG KPV76, see also Sch78.

There are several easy ways to see that an irreducible coregular representation fails to have the 2-polarization property. One of the following can occur:

(i) $V$ is a symplectic representation of $G$.

(ii) $\mathbb{C}[V]^{G}$ is minimally generated by homogeneous elements of degrees $m_{1}, \ldots, m_{k}$ where $\sum_{i}\left(m_{i}+1\right)<\operatorname{dim} \mathbb{C}[2 V]^{G}$ or $\sum_{i}\left(m_{i}+1\right)=\operatorname{dim} \mathbb{C}[2 V]^{G}$ and $(2 V, G)$ is not coregular.

(iii) $(V, G)$ is the adjoint representation of $G$ where $G$ has rank at least two. Here there is a slice representation whose effective part is the adjoint representation of $A_{2}, B_{2}$ or $G_{2}$. Then we can apply (ii).

Examples of (i) are the representations $\left(\varphi_{3}, A_{5}\right),\left(\varphi_{3}, C_{3}\right)$ and $\left(\varphi_{5}, B_{5}\right)$. The representations $\left(\varphi_{3}, A_{n}, n=6,7\right)$ are examples of (ii) as is $\left(\varphi_{1}, F_{4}\right)$. Now we mention the remaining irreducible coregular representations that can't be decided by the criteria above.

(a) $\left(\varphi_{8}, D_{8}\right)$. Here $\mathbb{C}[V]^{G}$ has generators in degree $2,8, \ldots$ while $\mathbb{C}[2 V]^{G}$ has three bihomogeneous invariants of degree $(2,2)$.

(b) $\left(\varphi_{3}, A_{8}\right)$. Here $\mathbb{C}[V]^{G}$ has generators in degrees $12,18, \ldots$ while $\mathbb{C}[2 V]^{G}$ has a bihomogeneous generator in degree $(3,3)$.

(c) $\left(\varphi_{1}^{2}, B_{n}\right), n \geq 2$ or $\left(\varphi_{1}^{2}, D_{n}\right), n \geq 3$. Up to a trivial factor, these are just the representations of the groups $\mathrm{SO}_{n}$ on $\mathrm{S}^{2}\left(\mathbb{C}^{n}\right), n \geq 5$. Let $e_{1}, \ldots e_{n}$ be the standard basis of $\mathbb{C}^{n}$. The slice representation at the point $e_{1}^{2}+\cdots+e_{n-3}^{2}$ is, up to trivial factors, the sum $\left(\mathrm{S}^{2}\left(\mathbb{C}^{n-3}\right), \mathrm{SO}_{n-3}\right) \oplus\left(\mathrm{S}^{2}\left(\mathbb{C}^{3}\right), \mathrm{SO}_{3}\right)$ and the latter representation does not have the 2-polarization property by (ii).

(d) $\left(\varphi_{2}, C_{n}\right), n \geq 3$. In case that $n=3$, the representation fails to have the 2-polarization property by (ii). For $n \geq 4$, the representations have a slice representation which contains a factor $\left(\varphi_{2}, C_{3}\right)$, similarly to case (c).

The remaining representations $\left(\varphi_{4}, A_{7}\right)$ and $\left(\varphi_{4}, C_{4}\right)$ are handled as in (a) and (b).

One can now use the tables of $\underline{\operatorname{Sch} 78}$ to see which irreducible representations have the $k$-polarization property for $k \geq 3$.

Corollary 4.4. Let $G$ be simple and $V$ irreducible with the $k$-polarization property, $k \geq 3$. Then $(V, G)$ and $k$ are on the following list.

(1) $\left(\varphi_{1}, A_{n}\right), n \geq 2$ and $3 \leq k<n+1$.

(2) $\left(\varphi_{1}, B_{n}\right), n \geq 2$ and $3 \leq k<2 n+1$.

(3) $\left(\varphi_{1}, D_{n}\right), n \geq 3$ and $3 \leq k<2 n$.

(4) $\left(\varphi_{3}, B_{3}\right)$ and $k=3$.

It is also easy to determine the reducible representations with the $k$-polarization property for $k \geq 2$. One uses Lemma 3.1 the criterion (ii) above and the tables of Sch78. 
Corollary 4.5. Let $V$ be the direct sum of at least two irreducible nontrivial $G$ modules, where $G$ is simple. If $V$ has the $k$-polarization property, $k \geq 2$, then, up to isomorphism, $G=\mathrm{SL}_{n}, n \geq 5$ and $V=j \mathbb{C}^{n}$ where $2 \leq j<n / k$.

\section{REFERENCES}

[DKW06] J. Draisma, G. Kemper, D. L. Wehlau, Polarization of Separating Invariants, to appear in J. Can. Math. Soc.

[Hun97] M. Hunziker, Classical invariant theory for finite reflection groups, Transform. Groups 2 (1997), 147-163.

[KPV76] V. G. Kac, V. I. Popov, E. B. Vinberg, Sur les groupes linéaires dont l'algèbre des invariants est libre, C. R. Acad. Sc. Paris 283 (1976), 875-878.

[KrWa04] H. Kraft, N. R. Wallach, Polarizations and nullcone of representations of reductive groups, unpublished preprint (2004).

[vL94] M. A. A. van Leeuwen, LiE, a software package for Lie group computations, Euromath Bull. 1 (1994), 83-94.

[LMP06] M. Losik, P. Michor, V. Popov, On polarizations in invariant theory, J. Algebra 301 (2006), 406-424.

[Lu73] D. Luna, Slices étales, Bull. Soc. Math. France, Mémoire 33 (1973), 81-105.

[Sch78] G. W. Schwarz, Representations of simple Lie groups with regular rings of invariants, Invent. Math. 49 (1978), 167-191.

[Wa193] Nolan R. Wallach, Invariant differential operators on a reductive Lie algebra and Weyl group representations, J. Amer. Math. Soc. 6 (1993), 779-816.

[Weyl46] H. Weyl, The Classical Groups, 2nd edn., Princeton Univ. Press, Princeton, 1946.

Gerald W. Schwarz

Department of Mathematics

BRANDEIS UNIVERSITY

PO Box 549110

Waltham, MA 02454-9110

E-mail address: schwarz@brandeis.edu 